\title{
LETTER
}

\section{Low-dose quercetin positively regulates mouse healthspan}

\section{Dear Editor,}

Aging is the leading risk factor for many chronic diseases, accounting for almost $60 \%$ of all deaths worldwide. How to achieve healthy aging, alleviate aging-related diseases, and extend healthspan has become a main topic of biomedical research (He et al., 2019). Geroprotective compounds, such as metformin and rapamycin, have been shown to improve both healthspan and lifespan in mice (Martin-Montalvo et al., 2013; Bitto et al., 2016), whereas nicotinamide partially improves healthspan in mice (Mitchell et al., 2018). In addition, senolytics, compounds that eliminate senescent cells, have been proven to improve physical function and increase lifespan in mice (Xu et al., 2018). Although none have proven to be clinically reliable in delaying aging or treating frailty in humans, these compounds have already provoked enthusiasm for identifying a potential "elixir". Therefore, the exploration of more geroprotective compounds, especially natural active compounds, holds great potential for the development of geriatric medicines.

Quercetin (Que) is a natural bioflavonoid found in fruits and vegetables such as apples and onions. Que $(50 \mathrm{mg} / \mathrm{kg})$ in combination with dasatinib $(5 \mathrm{mg} / \mathrm{kg}$ ) (abbreviated as D + $Q$ ) has been shown to effectively eliminate senescent cells via induction of apoptosis, thus alleviating senescence-related phenotypes and improving physical function and lifespan in mice (Zhu et al., 2015; Xu et al., 2018). In addition, Que $(10 \mathrm{mg} / \mathrm{kg})$ in combination with dasatinib $(5 \mathrm{mg} / \mathrm{kg})$ has been reported to reduce hepatic steatosis (Ogrodnik et al., 2017). In each of these in vivo studies, however, Que was used at high doses ranging from 10 to $50 \mathrm{mg} / \mathrm{kg}$ body weight, which raises concerns about dose-dependent side effects such as headaches and limb tingling (Shoskes et al., 1999). As a selective tyrosine kinase receptor inhibitor, dasatinib is associated with warnings and precautions including pulmonary arterial hypertension and low blood cell counts. Therefore, high-dose Que and extra side effects of dasatinib would hamper potential clinical applications of Que in geriatric medicines. Through natural products screening using Werner syndrome (WS) human mesenchymal stem cells (hMSCs), we recently identified Que as a geroprotective agent that counteracts accelerated and natural aging of hMSCs at a concentration of as low as $100 \mathrm{nmol} / \mathrm{L}$, which is
100 times lower than the concentration of Que $(10 \mu \mathrm{mol} / \mathrm{L})$ previously used in combination with dasatinib as senolytic drugs to eliminate senescent cells in human umbilical vein cells (HUVECs) (Zhu et al., 2015; Geng et al., 2018).

To explore the geroprotective effect of low-dose Que monotherapy in rodents, we evaluated the in vivo effect of long-term low-dose Que administration under physiologicalaging condition. Que was given to 14-month-old C57BL/6J male mice by weekly oral gavage at a concentration of 0.125 $\mathrm{mg} / \mathrm{kg}$ body weight, which is $80-400$ times lower than that of the previously tested $D+Q(10-50 \mathrm{mg} / \mathrm{kg}$ body weight) regimens (Fig. 1A), with vehicle (10\% PEG400 in PBS)treated mice as controls (Zhu et al., 2015; Xu et al., 2018). After eight months of treatment, Que-treated mice showed decreased hair loss with normal food intake, body weight, blood glucose and bone mineral density (Figs. 1B and S1AD). Compared to vehicle-treated mice, mice subjected to Que treatment showed markedly improved exercise endurance in the RotaRod and treadmill tests, but normal grip strength by grip strength meter assay (Figs. 1C, 1D, and S1E-G). Accordingly, the cardiac function of these mice was examined by Doppler tissue imaging. Although ejection fraction (EF) and fractional shortening (FS) were unaffected, a higher frequency of the mitral ratio of peak early to late diastolic filling velocity $(E / A)$ within the normal range was observed in Que-treated mice than in the age-matched controls (Figs. 1E and $\mathrm{S} 1 \mathrm{H}$ ). However, the lifespan was not prolonged by low-dose Que treatment observed up to the age of 31 months (Fig. S1I). Taken together, these data indicate that long-term low-dose Que administration alone sufficiently improves multiple aspects of healthspan, but not lifespan, in mice.

To investigate how Que improved healthspan in mice, we collected 11 different kinds of tissues from 10-week young male mice (Y-Ctrl) and vehicle (O-Veh)- and low-dose Quetreated 22-month old male mice (O-Que). No significant difference was observed in organ weights between O-Veh and O-Que (Fig. S2A). Given that exercise endurance and diastolic function were improved by Que, we particularly examined the changes in skeletal muscles (SKM), white adipose tissues (WAT), brown adipose tissues (BAT) and hearts. Upon Que treatment, the arrangement of muscle fibers became more regular and compact with less fibrosis 
A

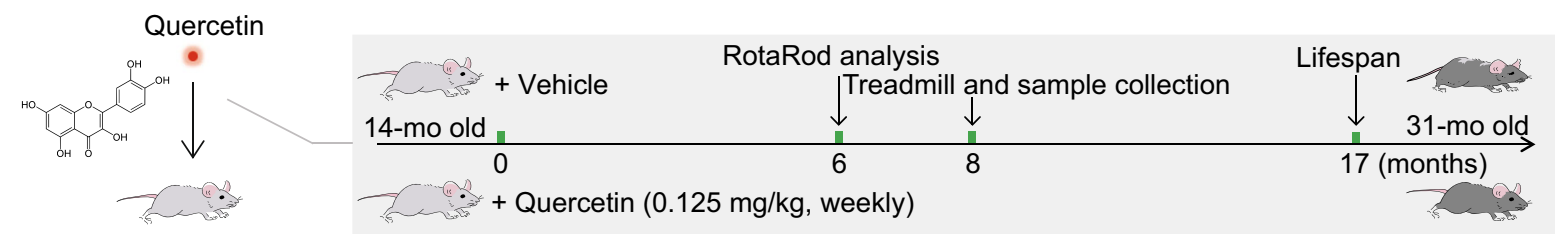

B

$\mathrm{E}$
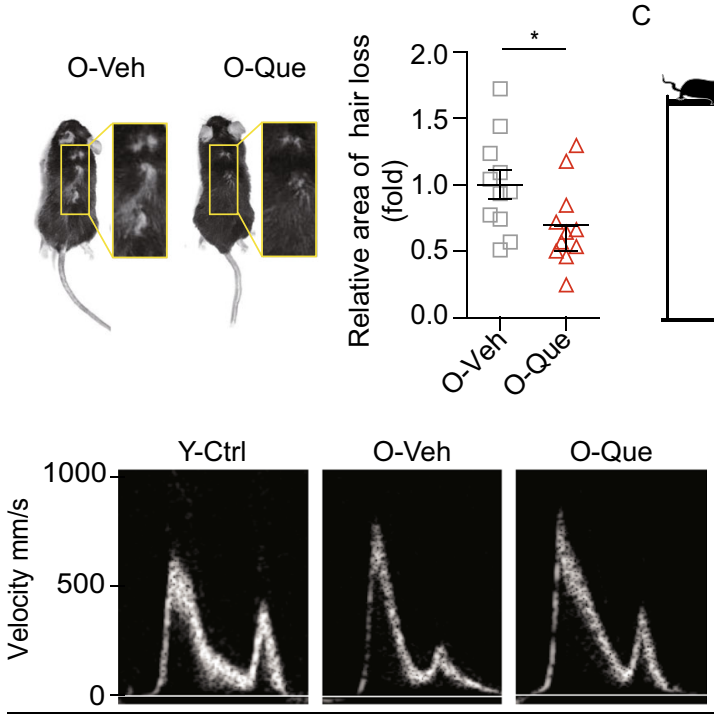

$\mathrm{E} / \mathrm{A}<1.3 \mathrm{E} / \mathrm{A}=1.3 \sim 2.5 \mathrm{E} / \mathrm{A}>2.5 \quad \mathrm{E} / \mathrm{A}=1.3 \sim 2.5$

\begin{tabular}{ccccc}
\multicolumn{2}{r}{ (Number) } & (Number) & (Number) & (Ratio) \\
\hline Y-Ctrl & 0 & 12 & 0 & $100 \%$ \\
O-Veh & 3 & 4 & 5 & $33.33 \%$ \\
O-Que & 4 & 8 & 0 & $66.67 \%$
\end{tabular}

G SKM

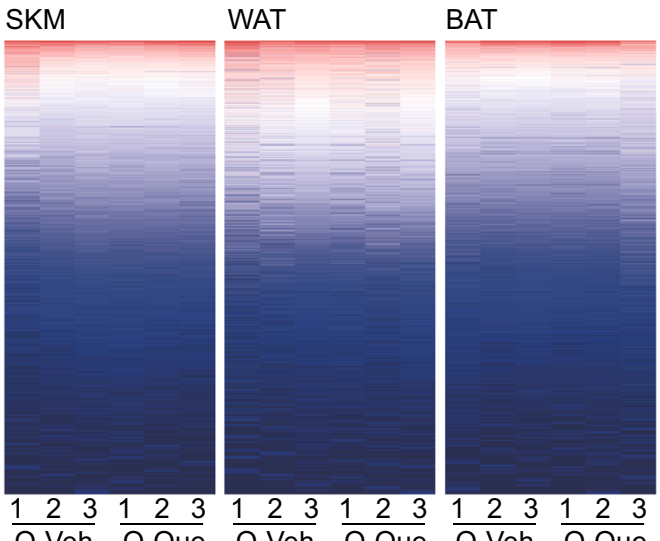

C
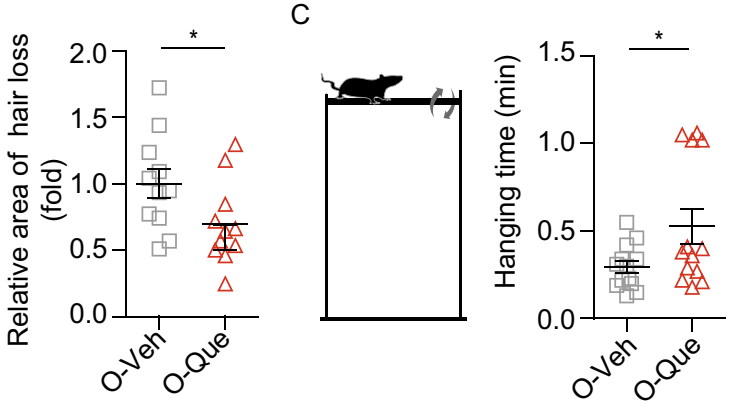

D

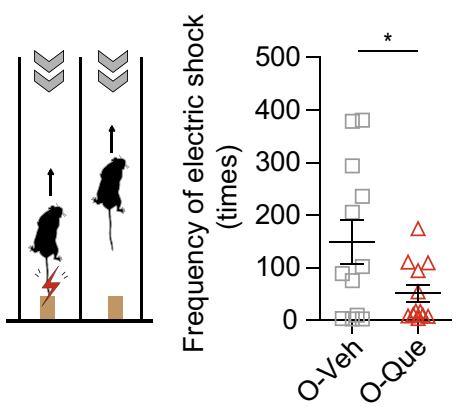

F
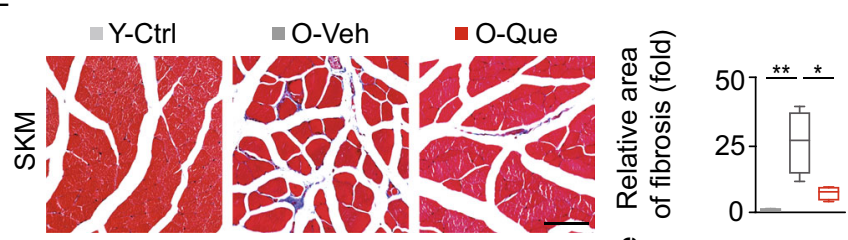

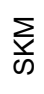

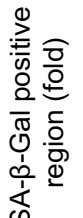

$80 * *$

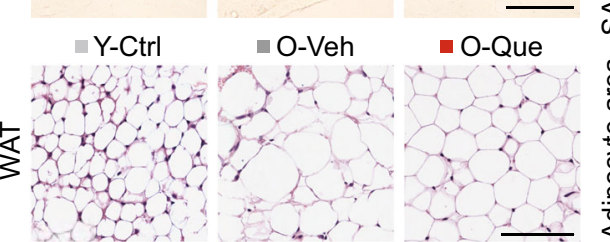

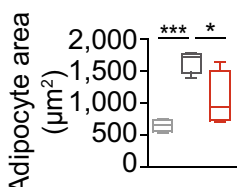

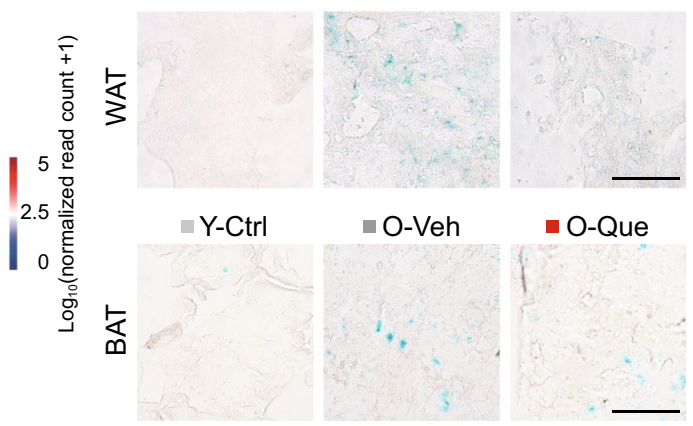

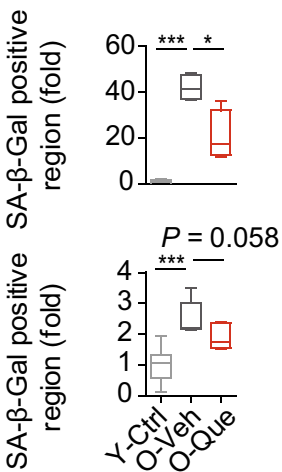


Figure 1. Low-dose quercetin alone improved the healthspan of physiologically aging mice. (A) Experimental design for drug administration. (B) Hair loss evaluation $(n=11)$. Data are shown as the mean \pm SEM. ${ }^{*} P<0.05$. (C) Hanging endurance on the RotaRod system $(n=13)$. Data are shown as the mean \pm SEM. ${ }^{*} P<0.05$. (D) Frequency of electric shock on the treadmill over $30 \mathrm{~min}(n=12)$. Data are shown as the mean \pm SEM. ${ }^{*} P<0.05$. (E) The ratio of peak velocity of early to late filling of mitral inflow $(E / A)(n=12)$. The table shows the number of mice in 3 kinds of $E / A$ ranges, and Que treatment increased the ratio of normal E/A mice. ${ }^{*} P<0.05$. (F) Masson's trichrome staining in SKM showed moderate perivascular and interstitial fibrosis (blue areas) $(n=4)$. Data are shown as the mean \pm SEM. * $P<0.05$. Scale bar, $100 \mu \mathrm{m}$. SA- $\beta-$ Gal staining analysis of SKM, WAT and BAT. Scale bar, $100 \mu \mathrm{m}(n=4)$. Data are shown as the mean \pm SEM. ${ }^{* * *} P<0.001,{ }^{* *} P<0.01$, ${ }^{*} P<0.05$. Haematoxylin and eosin staining of WAT. Scale bar, $100 \mu \mathrm{m}$ $(n=4)$. Data are shown as the mean \pm SEM. ${ }^{*} P<0.05$. (G) Global gene expression profiling in SKM, WAT and BAT $(n=$ 3). Y-Ctrl represents 10 -week-old young male mice, and O-Veh and O-Que represent vehicle (10\% PEG400 in PBS)- or lowdose Que-treated old male mice.

and senescence (Figs. 1F and S2B). In WAT, the increases in adipocyte size and senescence-associated $\beta$-galactosidase (SA- $\beta$-Gal)-positive area during aging were both alleviated upon Que treatment (Fig. 1F). In BAT, although adipocyte size was unaffected, there was a decreasing trend of the SA- $\beta$-Gal-positive area upon Que treatment (Figs. 1F and S2B). By comparison, we did not observe any significant differences in mouse hearts by histological analysis and SA$\beta$-Gal staining (Fig. S2B). Therefore, these data suggest that long-term low-dose Que administration may delay aging of SKM, WAT, and BAT in mice.

To further explore the molecular mechanisms of the beneficial effects of Que, we performed whole-transcriptome RNA sequencing (RNA-seq) of SKM, WAT, and BAT from Y-Ctrl, O-Veh, and O-Que mice. Global gene expression profiling revealed that most protein coding genes were unaffected after long-term low-dose Que administration (Fig. 1G). Accordingly, we inferred that low-dose Que might exert its senostatic effect by regulating the expression of non-protein-coding RNAs.

We previously observed that Que alleviates hMSC senescence in part through the restoration of heterochromatin architecture in prematurely and physiologically aging hMSCs (Geng et al., 2018). Constitutive heterochromatins are predominantly comprised of repetitive elements (REs), including retrotransposable elements (RTEs). The expression of RTEs is repressed via epigenetic regulation under normal conditions but is elevated during physiological aging, eliciting active transposition (De Cecco et al., 2013). Accordingly, mobilization of RTEs is likely to be a key contributor to tissue aging and cell degeneration (De Cecco et al., 2013). To investigate whether low-dose Que treatment antagonized the activation of RTEs, we examined the expression levels of various RTEs, including long terminal repeats such as LTR10C, LTR2C, LTR35A, and LTR3B, and non-long terminal repeats including long interspersed nuclear elements 1 (L1, also known as LINE-1) and short interspersed nuclear elements (SINEs) such as Alu in WS hMSCs after continuous Que treatment at the concentration of $100 \mathrm{nmol} / \mathrm{L}$. Que treatment silenced the transcription of various RTEs in WS hMSCs, consistent with the rejuvenated cellular phenotypes (Fig. 2A). To test whether Que treatment may also repress activation of RTEs in a mouse in vivo model, we compared the transcriptional levels of RTEs such as L1, SINE B1, LTR41, LTR42 and MLV5 in multiple tissues of Y-Ctrl, O-Veh, and O-Que mice. Consistently, most RTEs were transcriptionally upregulated in the SKM and BAT of old mice compared to those of young mice and were repressed by Que treatment (Fig. 2B). Similar to the tendency in the BAT, RTEs in WAT from Que-treated mice were also slightly decreased (Fig. 2B). In line with enhanced L1 transcripts, there was an increased expression level of $L 1$ open reading frame 1 protein (ORF1p) in SKM and BAT of aged mice, which could be reversed by long-term low-dose Que administration (Fig. 2C-E). These data indicate that Que represses RTE activation in senescent hMSCs and multiple aged mouse tissues.

In senescent cells, the activation of RTEs (such as L1) leads to genome instability and accumulation of cytosolic DNA that further binds to cytosolic sensor cGAS and activates TBK1 and IRF3, which subsequently promote senescence-associated secretory phenotype (SASP) (Takahashi et al., 2018; De Cecco et al., 2019). In addition, NF-kB/RelA in cGAS-STING-mediated NF-KB pathway acts with IRF3 and other transcription factors to induce the expression of inflammatory cytokines such as IL-6, the most prominent SASP cytokine (Chen et al., 2016). Notably, both p-TBK1 and $p$-IRF3 were increased in old mouse tissues compared to the young ones and were repressed upon Que treatment (Fig. 2E), indicating the effect of Que on inhibiting cGASSTING pathway (Kato et al., 2017). Similarly, RelA (p65) was upregulated in aged mouse tissues and repressed upon Que treatment (Fig. 2E-G). Consistently, the inflammatory cytokine IL-6 was increased in old mice compared to young mice and Que antagonized the increase of IL-6 in both WShMSCs and old mouse SKM and BAT (Fig. 2A and 2B). Thus, our data suggest that Que may block SASP through the axis of heterochromatin-RTEs (L1)-innate immune response pathway (Fig. $2 \mathrm{H}$ ).

In this study, we reported for the first time a geroprotective effect of low-dose quercetin alone that improved the healthspan of aged C57BL/6J male mice. Que-treated mice showed less hair loss, greater athletic endurance, enhanced diastolic function, and less muscle fibrosis, as well as alleviated cellular senescence in multiple tissues. Interestingly, these changes appear to be rarely associated with transcriptional alterations of protein-coding genes but are linked 


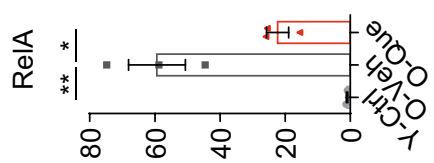

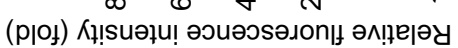

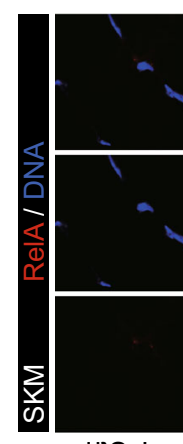

แศว-人

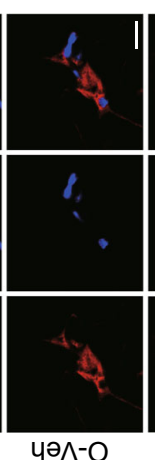

范 离

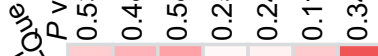
हैं हैं

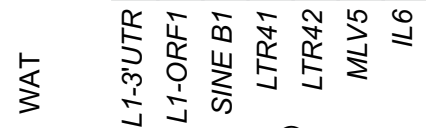

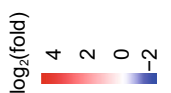

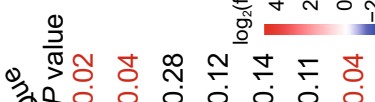

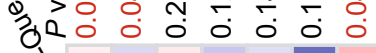
\% हैं।

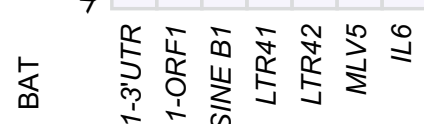
ปป क

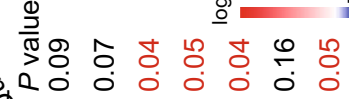

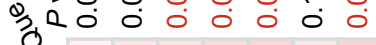
\%० त्रें

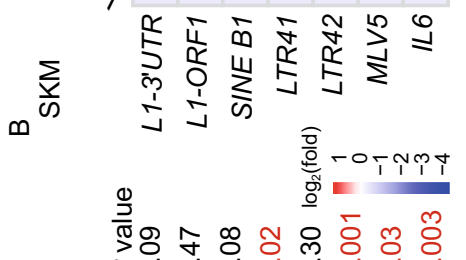

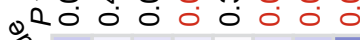

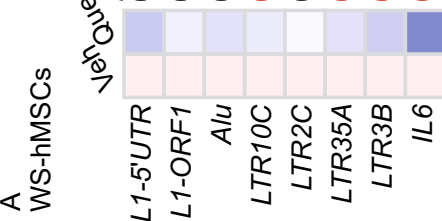

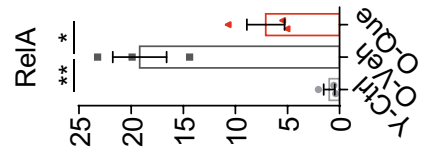

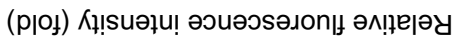

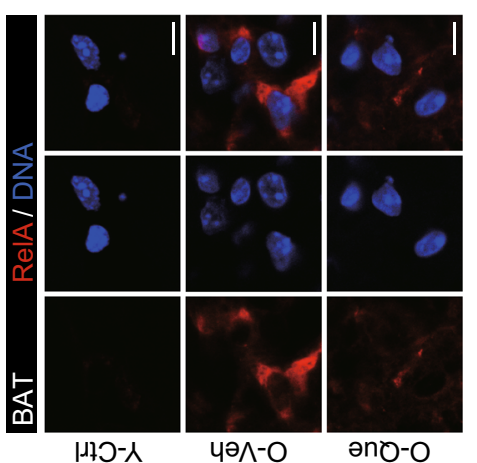

0
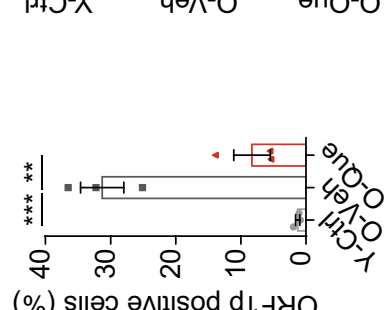

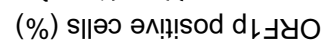

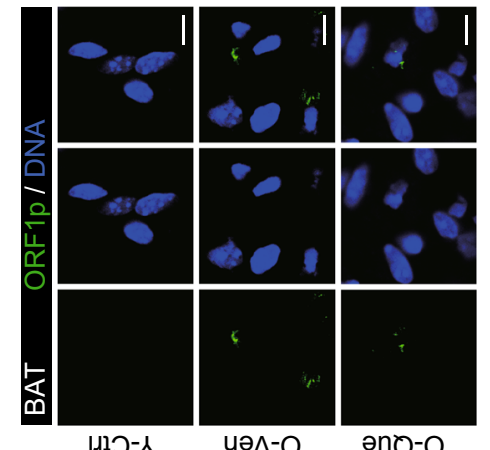

๑

|มว-入

əno-O

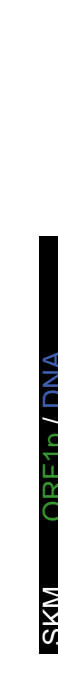

u

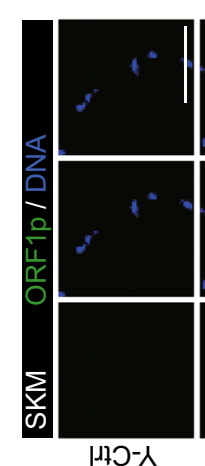

|ฌว-ᄉ

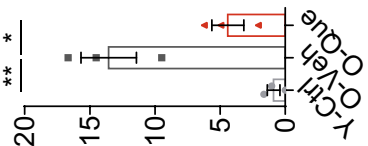

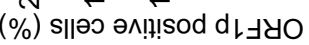

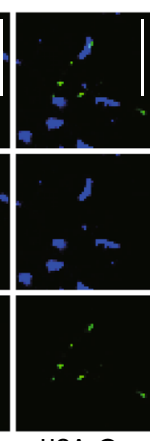

чә^-О

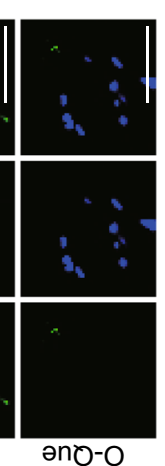

əno-o

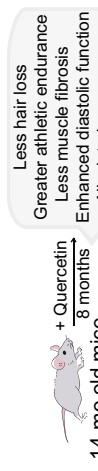

I

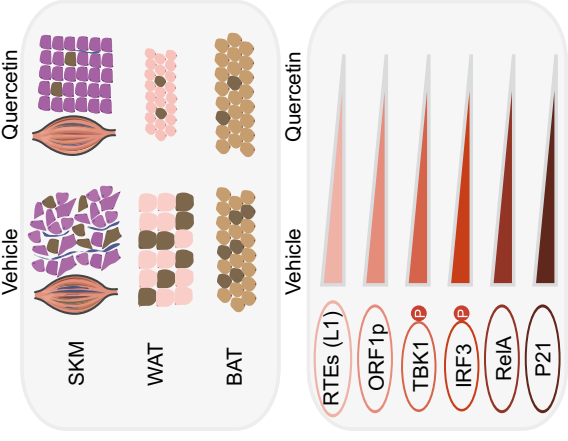

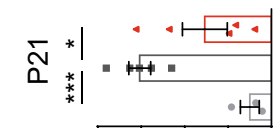

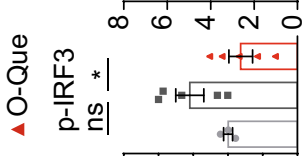
กั่

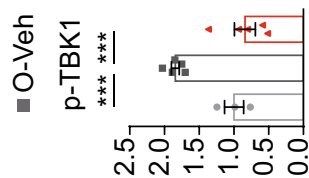

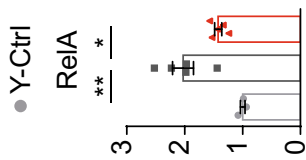

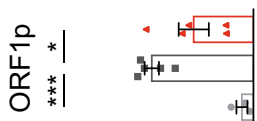

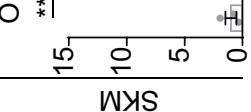

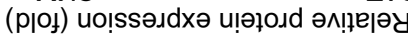

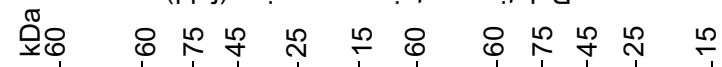

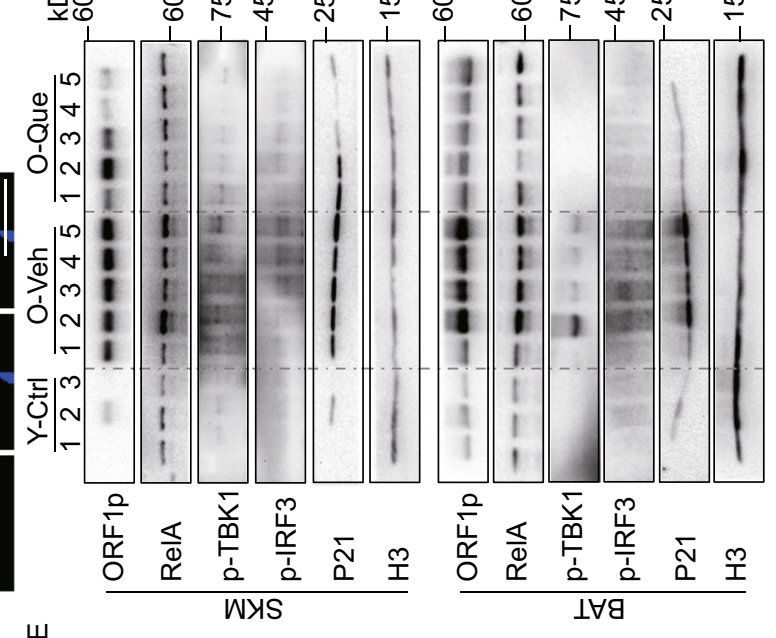


Figure 2. Activation of retrotransposable elements (RTEs) was repressed in Que-treated WS hMSCs and certain mouse tissues. (A) RT-qPCR analysis of RTEs in vehicleand Que-treated WS hMSCs (passage 7) $(n=3)$. $P$ values between vehicle and Que are shown on the right. (B) RT-qPCR analysis of RTEs in SKM, WAT and BAT of young male mice, old male mice treated with vehicle and Que $(n=4)$. $P$ values between $\mathrm{O}-\mathrm{Veh}$ and $\mathrm{O}-\mathrm{Que}$ are shown on the right, $P \leq 0.05$ were labeled in red. (C) Immunostaining of ORF1p in SKM of young male mice, old male mice treated with vehicle and Que $(n=3)$. Scale bar, $50 \mu \mathrm{m}$. Data are shown as the mean \pm SEM (cell number $\geq 100$ ). ${ }^{* *} P<0.01,{ }^{*} P<0.05$. (D) Immunostaining of ORF1 $\mathrm{p}$ in the BAT of young male mice, old male mice treated with vehicle and Que $(n=3)$. Scale bar, $7.5 \mu \mathrm{m}$. Data are shown as the mean \pm SEM (cell number $\geq 100$ ). ${ }^{* \star *} P<0.001$, ${ }^{\star *} P<$ 0.01. (E) Immunoblotting of ORF1p, RelA, p-TBK1, p-IRF3 and P21 in the SKM and BAT of young male mice $(n=3)$, old male mice treated with vehicle $(n=5)$ and Que $(n=5)$. ${ }^{* *} P<0.001$, ${ }^{* *} P<0.01,{ }^{*} P<0.05$, ns, not significant. (F) Immunostaining of RelA in the SKM of young male mice, old male mice treated with vehicle and Que $(n=3)$. Scale bar, $10 \mu \mathrm{m}$. Data are shown as the mean \pm SEM (cell number $\geq 100$ ). ${ }^{* *} P<0.01,{ }^{*} P<0.05$. (G) Immunostaining of RelA in the BAT of young male mice, old male mice treated with vehicle and Que $(n=3)$. Scale bar, 7.5 $\mu \mathrm{m}$. Data are shown as the mean \pm SEM (cell number $\geq 100$ ). ${ }^{* *} P<0.01,{ }^{*} P<0.05$. Y-Ctrl represents 10 -week-old young male mice, and O-Veh and O-Que represent vehicle (10\% PEG400 in PBS)- or low-dose Que-treated old male mice. (H) A proposed model illustrating the senostatic effects of Que.

to heterochromatin stabilization and RTE silencing. Que treatment prevented L1 from hyperactivation, thereby inhibiting SASP. In contrast to the reported senolytic effect of high-dose $D+Q$ (Xu et al., 2018), where Que exerts geroprotective effects via the induction of apoptosis of senescent cells, low-dose Que $(0.125 \mathrm{mg} / \mathrm{kg}$ body weight) alone was sufficient to exert senostatic effects in mice by affecting heterochromatin stability through repression of RTEs activity in this study. In a translational context, low-dose Que monotherapy may be helpful to minimize the dose-dependent side effects compared to high-dose administration and avoid drug interference when used in combination, probably representing a potential therapeutic option for future clinical application (Shoskes et al., 1999). Of note, here we reported the geroprotective effect of Que in male mice and its effect remains to be studied in female mice.

The possible mechanism of low-dose Que in mice may be associated with its function as a heterochromatin stabilizer and its direct inhibitory activity against reverse transcriptase
(Ono et al., 1990; Geng et al., 2018). Loss of heterochromatin architecture and genomic instability are two hallmarks of aging (Zhang et al., 2015). In advanced age, the expression of RTEs is often increased, which may in turn contribute to genomic instability and aging-associated cellular defects (De Cecco et al., 2013). Activation of L1 has been implicated in a variety of age-related disorders, including cancer and neurodegenerative diseases. The activation of L1 (and possibly other RTEs in mice) promotes the expression inflammatory factors, a feature of cellular senescence (De Cecco et al., 2019; Simon et al., 2019). Recently, it has been reported that nucleoside reverse-transcriptase inhibitors (NRTIs), such as lamivudine, stavudine, inhibit $\mathrm{L} 1$ retrotransposition and thus improve the healthspan and/or lifespan of SIRT6-knockout and physiologically aged mice (De Cecco et al., 2019; Simon et al., 2019). Que has been proven as a potential inhibitor of reverse transcriptase from Rauscher murine leukemia virus (RLV) and human immunodeficiency virus (HIV) by enzyme kinetic analysis, whereas its reverse transcriptase inhibition activity against RTEs in hMSCs and rodents has not been reported (Ono et al., 1990). Our data provide important evidence supporting the role of low-dose Que in safeguarding genomic stability (i.e. inhibition of retrotransposition), which at least in part contributes to its geroprotective activity in rodents.

\section{FOOTNOTES}

We thank Lei Bai, Jing Lu, Ying Yang, Qun Chu, Shikun Ma, and Ruijun Bai for administrative assistance. We thank Jingyi Jia (Xuan Wu Hospital), Sai Yang (IBP, CAS), Qi Wei (IBP, CAS), Mengfei Wang (IBP, CAS), and Xinyi Wu (IBP, CAS) for breeding and management of laboratory animals and Lei Zhou (IBP, CAS) for providing veterinary care. We thank Qing Xu (Capital Medical University) for Doppler tissue imaging. High-throughput sequencing data were processed on the "Era" petascale supercomputer of Computer Network Information Center of Chinese Academy of Sciences. This work was supported by the Strategic Priority Research Program of the Chinese Academy of Sciences (XDA16010100), the National Key Research and Development Program of China (2015CB964800, 2018YFC2000100, 2017YFA0102802, 2017YFA0103304, 2018YFA0107203), the National Natural Science Foundation of China (Grant Nos. 81625009, 81330008, 91749202, 91749123, 31671429, $81671377,81771515,31601158,81701388,81601233,81571385$, 31601109, 81822018, 81870228, 81801399, 31801010, 81801370, 81861168034), Beijing Municipal Commission of Health and Family Planning (PXM2018_026283_000002), Advanced Innovation Center for Human Brain Protection (3500-1192012) and the State Key Laboratory of Membrane Biology.

The authors declare no conflicts of interest. 
Lingling Geng ${ }^{1,2}$, Zunpeng Liu ${ }^{3,4,5}$, Si Wang ${ }^{2,6}$, Shuhui Sun ${ }^{2,6}$, Shuai Ma ${ }^{2,6}$, Xiaoqian Liu ${ }^{3,5}$, Piu Chan ${ }^{1}$, Liang Sun ${ }^{7}$, Moshi Song ${ }^{2,5,8 \bowtie}$, Weiqi Zhang ${ }^{4,5,8 凶}$, Guang-Hui Liu ${ }^{1,2,5,6,8,9 凶}$, Jing $\mathrm{Qu}^{3,5,8 \bowtie}$

${ }^{1}$ Advanced Innovation Center for Human Brain Protection, National Clinical Research Center for Geriatric Disorders, Xuanwu Hospital Capital Medical University, Beijing 100053, China

2 State Key Laboratory of Membrane Biology, Institute of Zoology, Chinese Academy of Sciences, Beijing 100101, China

${ }^{3}$ State Key Laboratory of Stem Cell and Reproductive Biology, Institute of Zoology, Chinese Academy of Sciences, Beijing 100101, China

${ }^{4}$ Key Laboratory of Genomics and Precision Medicine, Beijing Institute of Genomics, Chinese Academy of Sciences, Beijing 100101, China

${ }^{5}$ University of Chinese Academy of Sciences, Beijing 100049, China

${ }^{6}$ National Laboratory of Biomacromolecules, CAS Center for Excellence in Biomacromolecules, Institute of Biophysics, Chinese Academy of Sciences, Beijing 100101, China

7 The MOH Key Laboratory of Geriatrics, Beijing Hospital, National Center of Gerontology, Beijing 100730, China

${ }^{8}$ Institute for Stem Cell and Regeneration, Chinese Academy of Sciences, Beijing 100101, China

${ }^{9}$ Beijing Institute for Brain Disorders, Beijing 100069, China

$\triangle$ Correspondence: songmoshi@ioz.ac.cn (M. Song), weiqizhang@aliyun.com (W. Zhang), ghliu@ibp.ac.cn (G.-H. Liu), qujing@ioz.ac.cn (J. Qu)

\section{OPEN ACCESS}

This article is distributed under the terms of the Creative Commons Attribution 4.0 International License (http://creativecommons.org/ licenses/by/4.0/), which permits unrestricted use, distribution, and reproduction in any medium, provided you give appropriate credit to the original author(s) and the source, provide a link to the Creative Commons license, and indicate if changes were made.

\section{REFERENCES}

Bitto A, Ito TK, Pineda VV, LeTexier NJ, Huang HZ, Sutlief E, Tung H, Vizzini N, Chen B, Smith K et al (2016) Transient rapamycin treatment can increase lifespan and healthspan in middle-aged mice. eLife 5:e16351

De Cecco M, Criscione SW, Peterson AL, Neretti N, Sedivy JM, Kreiling JA (2013) Transposable elements become active and

Lingling Geng and Zunpeng Liu contributed equally to this work.

Electronic supplementary material The online version of this article (https://doi.org/10.1007/s13238-019-0646-8) contains supplementary material, which is available to authorized users. mobile in the genomes of aging mammalian somatic tissues. Aging 5:867-883

De Cecco M, Ito T, Petrashen AP, Elias AE, Skvir NJ, Criscione SW, Caligiana A, Brocculi G, Adney EM, Boeke JD et al (2019) L1 drives IFN in senescent cells and promotes age-associated inflammation. Nature 566:73-78

Geng L, Liu Z, Zhang W, Li W, Wu Z, Wang W, Ren R, Su Y, Wang P, Sun $L$ et al (2018) Chemical screen identifies a geroprotective role of quercetin in premature aging. Protein Cell 10(6):417-435

He X, Song M, Qu J, Guo Y, Cao H, Sun R, Liu G-H, Shen Y, Major Program Expert $G$ (2019) Basic and translational aging research in China: present and future. Protein Cell. https://doi.org/10.1007/ s13238-019-0617-0

Martin-Montalvo A, Mercken EM, Mitchell SJ, Palacios HH, Mote PL, Scheibye-Knudsen M, Gomes AP, Ward TM, Minor RK, Blouin MJ et al (2013) Metformin improves healthspan and lifespan in mice. Nat Commun 4:2192

Mitchell SJ, Bernier M, Aon MA, Cortassa S, Kim EY, Fang EF, Palacios HH, Ali A, Navas-Enamorado I, Di Francesco A et al (2018) Nicotinamide improves aspects of healthspan, but not lifespan, in mice. Cell Metab 27:667-676.e664

Ogrodnik M, Miwa S, Tchkonia T, Tiniakos D, Wilson CL, Lahat A, Day CP, Burt A, Palmer A, Anstee QM et al (2017) Cellular senescence drives age-dependent hepatic steatosis. Nat Commun 8:15691-15691

Ono K, Nakane H, Fukushima M, Chermann J-C, BarrÉ-Sinoussi F (1990) Differential inhibitory effects of various flavonoids on the activities of reverse transcriptase and cellular DNA and RNA polymerases. Eur J Biochem 190:469-476

Shoskes DA, Zeitlin SI, Shahed A, Rajfer J (1999) Quercetin in men with category III chronic prostatitis: a preliminary prospective, double-blind, placebo-controlled trial. Urology 54:960-963

Simon M, Van Meter M, Ablaeva J, Ke Z, Gonzalez RS, Taguchi T, De Cecco M, Leonova KI, Kogan V, Helfand SL et al (2019) LINE1 derepression in aged wild-type and SIRT6-deficient mice drives inflammation. Cell Metab 29:871-885.e875

Takahashi A, Loo TM, Okada R, Kamachi F, Watanabe Y, Wakita M, Watanabe S, Kawamoto S, Miyata K, Barber GN et al (2018) Downregulation of cytoplasmic DNases is implicated in cytoplasmic DNA accumulation and SASP in senescent cells. Nat Commun 9:1249

Xu M, Pirtskhalava T, Farr JN, Weigand BM, Palmer AK, Weivoda MM, Inman CL, Ogrodnik MB, Hachfeld CM, Fraser DG et al (2018) Senolytics improve physical function and increase lifespan in old age. Nat Med 24:1246-1256

Zhang W, Li J, Suzuki K, Qu J, Wang P, Zhou J, Liu X, Ren R, Xu X, Ocampo A et al (2015) A Werner syndrome stem cell model unveils heterochromatin alterations as a driver of human aging. Science (New York, NY) 348:1160-1163

Zhu Y, Tchkonia T, Pirtskhalava T, Gower AC, Ding H, Giorgadze N, Palmer AK, Ikeno Y, Hubbard GB, Lenburg M et al (2015) The Achilles' heel of senescent cells: from transcriptome to senolytic drugs. Aging Cell 14:644-658 